\title{
A LIMIT THEOREM FOR MEASURABLE RANDOM PROCESSES AND ITS APPLICATIONS
}

\author{
L. S. GRINBLAT
}

\begin{abstract}
Let the measurable random processes $\xi_{1}(t), \ldots, \xi_{n}(t), \ldots$ and $\xi(t)$ be defined on $[0,1]$. There exists $C$ such that for all $n$ and $t$ we have $E\left|\xi_{n}(t)\right|^{p} \leqslant C, p>1$. The following assertion is valid: if for any finite set of points $t_{1}, \ldots, t_{k} \subset[0,1]$ the joint distribution of $\xi_{n}\left(t_{1}\right), \ldots, \xi_{n}\left(t_{k}\right)$ converges to the joint distribution of $\xi\left(t_{1}\right), \ldots, \xi\left(t_{k}\right)$, and if $E\left|\xi_{n}(t)\right|^{p} \rightarrow E|\xi(t)|^{p}$ for all $t \in[0,1]$, then for any continuous functional $f$ on $L_{p}[0,1]$ the distribution of $f\left(\xi_{n}(t)\right)$ converges to the distribution of $f(\xi(t))$. This statement immediately implies the convergence of distributions in some limit theorems for the sums of independent random variables (for example, in one of the theorems of P. Erdös and M. Kac) and in some statistical criteria (for example, in the $\omega^{2}$-criterion of Cramér and von Mises).
\end{abstract}

1. Let $X$ be a metric space, and let $B$ be the $\sigma$-algebra of the Borel sets in $X$. The sequence of the probability measures $\mu_{n}$ on $B$ is said to be weakly converging to the measure $\mu$ if for any continuous function $f$ the distributions of $f$ with respect to $\mu_{n}$ converge to the distribution of $f$ with respect to $\mu$. The set $M$ of probability measures $\{\mu\}$ on $B$ is said to be weakly compact if for any sequence of measures $\mu_{n} \in M$ there exists a weakly convergent subsequence. The following is a theorem of Yu. V. Prohorov (see [4, IX, §1]).

TheOREM 1. Let $X$ be a metric space and let $M$ be the set of probability measures on $B$. If for any $\varepsilon>0$ there exists a compact subset $K$ such that $\sup \{\mu(X \backslash K) ; \mu \in M\}<\varepsilon$, then $M$ is a weakly compact set.

We shall use the following theorem of Fréchet and Kolmogoroff (see [5, IX, §1]).

THEOREM 2. A closed set $K \subset L_{p}[0,1]$ is compact if and only if:

(1) There exists $A$ such that $\int_{0}^{1}|x(t)|^{p} \leqslant A$ for all $x(t) \in K$;

(2) For any $\varepsilon>0$ there exists $\delta>0$ such that if $|\tau|<\delta$ then

$$
\int_{0}^{1}|x(t+\tau)-x(t)|^{p} d t<\varepsilon
$$

for all $x(t) \in K$, where the addition $t+\tau$ is considered modulo 1 .

2. Let $\xi(t, \omega)$ be a random process defined on $[0,1]$. This means that the probability space $\Omega=\{\omega\}$ and the probability $P$ on $\Omega$ are given, and for each

Received by the editors January 12, 1976.

AMS (MOS) subject classifications (1970). Primary 60B 10. 
$t$ the random variable $\xi(t, \omega)$ on $\Omega$ is defined. Consider the measure $\mu_{0}$ defined on the product of $\Omega$ and the interval $[0,1]$ which is the product of the probability $P$ and the Lebesgue measure on $[0,1]$. Following Doob, we say that the random process $\xi(t, \omega)$ is measurable if the function $\xi(t, \omega)$ is measurable with respect to $\mu_{0}$. We confine ourselves to random processes satisfying $E|\xi(t)|^{p} \leqslant C$ for all $t \in[0,1], p \geqslant 1$. In this case, the paths of $\xi(t, \omega)$ belong to $L_{p}[0,1]$ with probability 1 , and for any continuous functional $f$ on $L_{p}[0,1]$ the functional of the random process $f(\xi(t))$ is a random variable.

THEOREM 3. Let the measurable random processes $\xi_{1}(t), \ldots, \xi_{n}(t), \ldots$ and $\xi(t)$ be defined on $[0,1]$, and let there exist a member $C$ such that for all $n$ and $t$ we have $E\left|\xi_{n}(t)\right|^{p} \leqslant C, p \geqslant 1$. If for any finite set of points $t_{1}, \ldots, t_{k} \subset[0,1]$ the joint distribution of $\xi_{n}\left(t_{1}\right), \ldots, \xi_{n}\left(t_{k}\right)$ converges to the joint distribution of $\xi\left(t_{1}\right), \ldots, \xi\left(t_{k}\right)$, and if $E\left|\xi_{n}(t)\right|^{p} \rightarrow E|\xi(t)|^{p}$ for all $t \in[0,1]$, then for any continuous functional $f$ on $L_{p}[0,1]$ the distribution of $f\left(\xi_{n}(t)\right)$ converges to the distribution of $f(\xi(t))$.

Proof. We will denote a random process $\xi(t)$ by $\xi(t, \omega)$, with $\Omega=\{\omega\}$ a probability space. From the measurability of $\xi(t, \omega)$ it follows that for all $\varepsilon>0$ there exist mutually exclusive $\hat{\Omega}_{1}, \ldots, \hat{\Omega}_{r}$, points $0=d_{0}<d_{1}$ $<\cdots<d_{r}=1$, and a function $\hat{\xi}(t, \omega)$ such that

(1) $\Omega=\cup_{i=1}^{r} \hat{\Omega}_{i}$;

(2) The function $\hat{\xi}(t, \omega)$ is constant on sets of the form $\hat{\Omega}_{i} \times D_{j}, D_{j}=\{t$ : $\left.d_{j-1}<t<d_{j}\right\}$

(3) $\int|\xi(t, \omega)-\hat{\xi}(t, \omega)|^{p} d \mu_{0}<\varepsilon$, with $\mu_{0}$ as defined above.

Given $\varepsilon>0$ sufficiently small, let $[a, b] \subset[0,1]$ and set

$$
T_{\varepsilon}^{[a, b]}=\left\{t \in[a, b]: E|\xi(a)-\xi(t)|^{p} \leqslant \varepsilon\right\} .
$$

From the ideas which were introduced previously, it follows that there exists a sample of points $a_{1}<b_{1}<a_{2}<b_{2}<\ldots<a_{k}<b_{k} \subset[0,1]$ such that $\nu\left(T_{\varepsilon}^{\left[a_{i}, b_{i}\right]}\right)>(1-\varepsilon)\left(b_{i}-a_{i}\right)$ and $1-\sum_{i=1}^{k}\left(b_{i}-a_{i}\right)<\varepsilon$, where $\nu$ is the Lebesgue measure on $[0,1]$. From the condition $E\left|\xi_{n}(t)\right|^{p} \rightarrow E|\xi(t)|^{p}$ for all $t \in[0,1]$, we obtain that the function $\varphi_{n}^{i}(t)=E\left|\xi_{n}\left(a_{i}\right)-\xi_{n}(t)\right|^{p}$ converges pointwise on $[0,1]$ to the function $\varphi^{i}(t)=E\left|\xi\left(a_{i}\right)-\xi(t)\right|^{p}$. There exist sets $S_{\varepsilon}^{\left[a_{i}, b_{i}\right]} \subset T_{\varepsilon}^{\left[a_{i}, b_{i}\right]}$ and a positive integer $N$ such that

(1) $\varphi_{n}^{i}(t) \leqslant 2 \varepsilon$ if $n \geqslant N$ and $t \in S_{\varepsilon}^{\left[a_{i}, b_{i}\right]}$;

(2) $\nu\left(S_{\varepsilon}^{\left[a_{i}, b_{i}\right]}\right) \geqslant(1-2 \varepsilon)\left(b_{i}-a_{i}\right)$.

For $n \geqslant N$ we have

$$
\begin{aligned}
E \int_{a_{i}}^{b_{i}}\left|\xi_{n}\left(a_{i}\right)-\xi_{n}(t)\right|^{L} d t & \leqslant 2 \varepsilon \cdot \nu\left(S_{\varepsilon}^{\left[a_{i} b_{i}\right]}\right)+2^{p} \cdot C \cdot 2 \varepsilon \cdot\left(b_{i}-a_{i}\right) \\
& <2^{p+1} \cdot \varepsilon \cdot\left(b_{i}-a_{i}\right)(1+C),
\end{aligned}
$$

and thus

$$
E \sum_{i=1}^{k} \int_{a_{i}}^{b_{i}}\left|\xi_{n}\left(a_{i}\right)-\xi_{n}(t)\right|^{p} d t<2^{p+1} \cdot \varepsilon \cdot(1+C) .
$$


Suppose that the random variables $\xi_{n}(t)$ are defined on the probability space $\Omega=\{\omega\}$. For $n \geqslant N$ consider the set

$$
\Omega^{n}=\left\{\omega: \sum_{i=1}^{k} \int_{a_{i}}^{b_{i}}\left|\xi_{n}\left(\omega, a_{i}\right)-\xi_{n}(\omega, t)\right|^{p} d t<\vee \varepsilon\right\} .
$$

Obviously,

$$
P\left(\Omega^{n}\right)>1-2^{p+1} \cdot \vee \varepsilon \cdot(1+C) .
$$

Given $\delta>0$ such that $4 \delta<b_{i}-a_{i}$ for all $i$ and $4 \delta k<\varepsilon$. Consider the set

$$
\begin{aligned}
Q= & {\left[0, a_{1}+2 \delta\right] \cup\left[b_{1}-2 \delta, a_{2}+2 \delta\right] } \\
& \cup \cdots \cup\left[b_{i}-2 \delta, a_{i+1}+2 \delta\right] \cup \cdots \cup\left[b_{k}-2 \delta, 1\right] .
\end{aligned}
$$

Obviously, $\nu(Q)<2 \varepsilon$, and thus

$$
E \int_{Q}\left|\xi_{n}(t)\right|^{p} d t<2 \varepsilon \cdot C
$$

Consider the set $\Omega_{n}^{\prime}=\left\{\omega: \int_{Q}\left|\xi_{n}(\omega, t)\right|^{p} d t<\vee \varepsilon\right\}$. Obviously,

$$
P\left(\Omega_{n}^{\prime}\right)>1-2 \vee \varepsilon \cdot C \text {. }
$$

Consider the set $\Omega_{n}=\Omega^{n} \cap \Omega_{n}^{\prime}$. Obviously,

$$
P\left(\Omega_{n}\right)>1-2^{p+1} \cdot \vee \varepsilon \cdot(1+C)-2 \cdot \vee \varepsilon \cdot C .
$$

Consider the set

$$
\begin{aligned}
Q^{\prime}= & {\left[0, a_{1}+\delta\right] \cup\left[b_{1}-\delta, a_{2}+\delta\right] \cup \cdots \cup\left[b_{i}-\delta, a_{i+1}+\delta\right] } \\
\cup & \cdots \cup\left[b_{k}-\delta, 1\right] .
\end{aligned}
$$

If $\omega \in \Omega_{n}, n \geqslant N$, and $|\tau| \leqslant \delta$, then

$$
\begin{aligned}
\int_{0}^{1} \mid \xi_{n}(\omega, t+ & \tau)-\left.\xi_{n}(\omega, t)\right|^{p} d t \\
= & \sum_{i=1}^{k} \int_{a_{i}+\delta}^{b_{i}-\delta}\left|\xi_{n}(\omega, t+\tau)-\xi_{n}\left(\omega, a_{i}\right)+\xi_{n}\left(\omega, a_{i}\right)-\xi_{n}(\omega, t)\right|^{p} d t \\
& +\int_{Q}\left|\xi_{n}(\omega, t+\tau)-\xi_{n}(\omega, t)\right|^{p} d t \\
\leqslant & 2^{p} \cdot \sum_{i=1}^{k} \int_{a_{i}}^{b_{i}}\left|\xi_{n}\left(\omega, a_{i}\right)-\xi_{n}(\omega, t)\right|^{p} d t+2^{p} \cdot \int_{Q}\left|\xi_{n}(\omega, t)\right|^{p} d t \\
< & 2^{p+1} \cdot \sqrt{ } \varepsilon .
\end{aligned}
$$

The random processes $\xi_{1}(t), \ldots, \xi_{n}(t), \ldots$ define the probability measures $\mu_{1}, \ldots, \mu_{n}, \ldots$ on the Borel subset of $L_{p}[0,1]$. Consider the set in $L_{p}[0,1]$,

$$
K_{\varepsilon, \delta}=\left\{x(t): \int_{0}^{1}|x(t+\tau)-x(t)|^{p} d t<2^{p+1} \cdot \sqrt{ } \varepsilon \quad \text { if }|\tau| \leqslant \delta\right\} .
$$

For $n \geqslant N$ we have

$$
\mu_{n}\left(K_{\varepsilon, \delta}\right)>1-2^{p+1} \cdot \vee \varepsilon \cdot(1+C)-2 \cdot \vee \varepsilon \cdot C .
$$


There exists $\delta^{\prime}>0$ such that

$$
\mu_{n}\left(K_{\varepsilon, \delta^{\prime}}\right)>1-2^{p+1} \cdot \sqrt{ } \varepsilon \cdot(1+C)-2 \cdot \sqrt{ } \varepsilon \cdot C
$$

for all $n$. Hence, by virtue of the Fréchet-Kolmogoroff theorem for any $\varepsilon_{0}>0$ there exists a compact set $K \subset L_{p}[0,1]$ such that $\sup _{n} \mu_{n}\left(L_{p}[0,1] \backslash K\right)$ $<\varepsilon_{0}$. In view of Prohorov's theorem the statement of Theorem 3 is proved.

3. Let $\chi_{1}, \ldots, \chi_{n}, \ldots$ be a sequence of independent, equally distributed random variables, $E \chi_{n}=0, D \chi_{n}=1$. Set $S_{k}=\chi_{1}+\cdots+\chi_{k}$. One of the limit theorems of P. Erdös and M. Kac states (see [2]) that

$$
\lim _{n \rightarrow \infty} P\left\{\frac{S_{1}^{2}+\cdots+S_{n}^{2}}{n^{2}}<\alpha\right\}=\sigma(\alpha),
$$

where

$$
\sigma(\alpha)=\frac{\pi^{-3 / 2}}{4} \int_{0}^{\alpha / 2} d u u^{-3 / 2} \int_{0}^{\pi / 2}(\cos t)^{-1 / 2} \theta^{\prime}\left(t / 2, e^{-1 / 4 u}\right) d t \quad(\alpha \geqslant 0)
$$

and

$$
\theta(z, q)=2 \sum_{n=0}^{\infty}(-1)^{n} q^{(2 n+1)^{2} / 4} \sin (2 n+1) z, \quad \theta^{\prime}=\frac{\partial \theta}{\partial z} .
$$

Consider the random process

$$
W_{n}(t)=\frac{S_{k}}{\sqrt{ } n} \quad \text { if } t \in\left[\frac{k-1}{n}, \frac{k}{n}\right), \quad W_{n}(1)=\frac{S_{n}}{\sqrt{ } n}
$$

on $[0,1]$. Let $\mathscr{W}(t)$ be the Brownian motion process defined on $[0,1]$. The random processes $W_{n}(t)$ and $\mathcal{W}(t)$ satisfy all the conditions of Theorem 3 $(p=2)$ and thus we have

THEOREM 4. For any continuous functional $f$ on $L_{2}[0,1]$ the distribution of $f\left(W_{n}(t)\right)$ converges to the distribution of $f(\mathcal{W}(t))$.

In particular,

$$
\lim _{n \rightarrow \infty} P\left\{\int_{0}^{1} W_{n}^{2}(t) d t<\alpha\right\}=P\left\{\int_{0}^{1} \mathscr{W}(t) d t<\alpha\right\} .
$$

But

$$
\frac{S_{1}^{2}+\cdots+S_{n}^{2}}{n^{2}}=\int_{0}^{1} W_{n}^{2}(t) d t .
$$

Hence, Theorem 3 implies immediately the convergence of the distributions in the theorem of P. Erdös and M. Kac. R. Cameron and W. Martin proved (see [1]) that

$$
\sigma(\alpha)=P\left\{\int_{0}^{1} \mho^{2}(t) d t<\alpha\right\}
$$

Remark. The general limit theorems were considered by M. Donsker and Yu. V. Prohorov (see [4, IX, §2]). Nevertheless the fact that Theorem 4 
follows from such simple and general considerations is rather interesting.

4. Suppose that the results of some experiment form a random variable with the continuous distribution function $F(x)$. Suppose also that the results of $n$ independent tests are known: $\xi_{1}, \ldots, \xi_{n}$. Consider the empirical distribution function

$$
F_{n}^{*}(x)=\nu_{n}(x) / n,
$$

where $\nu_{n}(x)$ is the number of variables $\xi_{k}$ in the interval $(-\infty, x)$. The $\omega^{2}$-criterion states that

$$
\lim _{n \rightarrow \infty} P\left\{n \int_{-\infty}^{\infty}\left[F_{n}^{*}(x)-F(x)\right]^{2} d F(x)<\alpha\right\}=G(\alpha),
$$

where $G(\alpha)$ is the distribution function with characteristic function $(\sqrt{2 i \alpha} \csc \sqrt{2 i \alpha})^{1 / 2}$. Consider the random process $\eta_{n}(t)=$ $\eta_{n}^{\prime}\left(F^{-1}(t)\right)$ on $[0,1]$, where $\eta_{n}^{\prime}(x)=\sqrt{n}\left(F_{n}^{*}(x)-F(x)\right)$. Let $\eta(t)$ be a Gaussian process on $[0,1]$ such that $E \eta(t)=0, E \eta(t) \eta(s)=t(1-s)$ for $t<s$. The processes $\eta_{n}(t)$ and $\eta(t)$ satisfy the conditions of Theorem $3(p=2)$. Thus we have

THEOREM 5. For any continuous functional $f$ on $L_{2}[0,1]$ the distribution of $f\left(\eta_{n}(t)\right)$ converges to the distribution of $f(\eta(t))$.

In particular,

$$
\lim _{n \rightarrow \infty} P\left\{\int_{0}^{1} \eta_{n}^{2}(t) d t<\alpha\right\}=P\left\{\int_{0}^{1} \eta^{2}(t) d t<\alpha\right\} .
$$

But

$$
\int_{0}^{1} \eta_{n}^{2}(t) d t=n \int_{-\infty}^{\infty}\left[F_{n}^{*}(x)-F(x)\right]^{2} d F(x) .
$$

Hence, the convergence of distributions in $\omega^{2}$-criterion is proved. One can alsc show (see [4, IX, §8]) that

$$
G(\alpha)=P\left\{\int_{0}^{1} \eta^{2}(t) d t<\alpha\right\} .
$$

Remark. Concerning the various statistical criteria the reader is referred to [3], which also contains a rich bibliography on the subject. In [4, IX, §8] one can find the theorem that for any functional $f$, continuous in the Skorohod metric, the distribution of $f\left(\eta_{n}(t)\right)$ converges to the distribution of $f(\eta(t))$. Our Theorem 5 is a special case of this theorem. Nevertheless the fact that Theorem 5 follows from such simple and general considerations is rather interesting.

\section{REFERENCES}

1. R. H. Cameron and W. T. Martin, The Wiener measure of Hilbert neighborhoods in the space 
of real continuous functions, J. Math. Physics Mass. Inst. Tech. 23 (1944), 195-209. MR 6, 132.

2. P. Erdös and M. Kac, On certain limit theorems of the theory of probability. Bull. Amer. Math. Soc. 52 (1946), 292-302. MR 7, 459.

3. D. A. Darling, The Kolmogorov-Smirnov, Cramer-von Mises tests, Ann. Math. Statist. 28 (1957), 823-838. MR 20 \#390.

4. I. I. Gihman and A. V. Skorohod, Introduction to the theory of random processes, "Nauka", Moscow, 1965; English transl., Saunders, Philadelphia, Pa., 1969. MR 33 \#6689; 40 \#923.

5. K. Yosida, Functional analysis, Grundlehren math. Wiss., Band 123, Academic Press, New York; Springer-Verlag, Berlin, 1965. MR 31 \#5054.

Department of Mathematics, Bar-Ilan University, Ramat-Gan, IsRael 